Compr ehensi ve magnet ohydr odynami c hybri d si mul at $i$ ons of Al $f$ ven ei genmode burst $s$ and fast-i on I osses in the Large Hel i cal Devi ce

\begin{tabular}{|l|l|}
\hline $\begin{array}{l}\text { j our nal or } \\
\text { publ i cat i on } \mathrm{t} \text { i t l e }\end{array}$ & Nucl ear Fusi on \\
\hline vol une & 59 \\
\hline number & 9 \\
\hline page r ange & 096018 \\
\hline year & $2019-07-23$ \\
\hline URL & ht t p: //hdl . handl e. net /10655/00012894 \\
\hline
\end{tabular}




\title{
Comprehensive magnetohydrodynamic hybrid simulations of Alfvén eigenmode bursts and fast-ion losses in the Large Helical Device
}

\author{
R. Seki ${ }^{1,2}$, Y. Todo ${ }^{1}$, Y. Suzuki ${ }^{1,2}$, D.A. Spong ${ }^{3}$, K. Ogawa ${ }^{1,2}$, M. Isobe ${ }^{1,2}$, and M. Osakabe ${ }^{1,2}$ \\ ${ }^{1}$ National Institute for Fusion Science, National Institutes of Natural Sciences, Toki, Japan. \\ ${ }^{2}$ SOKENDAI (The Graduate University for Advanced Studies), Toki, Japan. \\ ${ }^{3}$ Oak Ridge National Laboratory, Oak Ridge, Tennessee 37831, USA
}

\begin{abstract}
Comprehensive magnetohydrodynamic (MHD) hybrid simulations with neutral beam injection and collisions were conducted to investigate the Alfvén eigenmode (AE) bursts and the fast-ion losses in the Large Helical Device (LHD) for the realistic conditions close to the experiments. It is found in the simulation of the slowing-down time scale that the $\mathrm{AE}$ bursts take place repetitively accompanied by fast-ion redistribution and losses leading to lower saturation levels of stored fast-ion energy than those in a classical calculation where the MHD perturbations are neglected. The fast-ion loss rate caused by the $\mathrm{AE}$ burst has the quadratic dependence on $\mathrm{AE}$ amplitude, which was observed in the LHD experiment. The majority of the lost fast ions are counter-passing particles whose velocity and pitch-angle are close to those of the beam injection.
\end{abstract} The second component of the lost fast ions is transit particles whose velocity is close to thermal 
velocity. The loss of the counter-passing particles occurs mainly during the AE bursts, while the transit particles are lost both during the $\mathrm{AE}$ bursts and the quiescent periods with larger loss rate than that in the classical calculation. The initial location of the lost counter-injected particles spreads from the plasma edge to the plasma center, while only the particles initially located in the peripheral region are lost for the co-injected beam.

\section{Introduction}

The evaluation of fast-ion confinement is indispensable for the prediction of the heating efficiency in fusion reactor. The fast-ion confinement depends not only on the collisional transport in the equilibrium magnetic field but also on the fast-ion driven instabilities such as Alfvén Eigenmondes (AE) which induce the fast-ion losses and the transport. Therefore, it is an important issue to identify the fast-ion driven instabilities and clarify the properties of the lost fast-ions due to the instabilities.

The studies of the fast-ion driven instabilities and the fast-ion loss process in tokamaks have been carried out experimentally and theoretically over the last two decades [1-3]. The relationship between the fast-ion loss and the amplitude of the AE instabilities was investigated [4-5]. 
The physics of AEs have been extensively studied in heliotron/stellarator devices with non-axisymmetric three-dimension magnetic configuration for systematic understanding of the AEs and the fast-ion loss process. There were observations of AE-induced or energetic-particle continuum mode (EPM)-induced losses in the Compact Helical System (CHS) [6] and global Alfvén eigenmode (GAE)-induced loss in the Wendelstein 7-AS [7]. In the Large Helical Device (LHD), which is one of the largest helical devices, the fast-ion driven instabilities such as the toroidal Alfvén eigenmondes (TAEs) were observed [8]. In addition, the AE-induced fast-ion losses were measured by a scintillator-based lost fast-ion probe. The relationship between amplitude of the instabilities and fast-ion losses were investigated[9].

On the other hand, since there is a toroidal dependence of fast-ion loss in the LHD even with no $\mathrm{AE}$ instabilities, it is difficult to achieve an overall understanding of fast-ion loss process only by the local measurements. Computer simulation is a powerful tool for investigating the interaction between fast-ions and fast-ion driven $\mathrm{AE}$ instabilities such as the fast-ion losses induced by the AEs. A hybrid simulation code for nonlinear magnetohydrodynamics (MHD) and energetic-particle dynamics, MEGA, has been developed to simulate recurrent bursts of fast-ion driven AE instabilities including the energetic-particle source, collisions, and losses in a non-axisymmetric three-dimension magnetic configuration such as the LHD[10]. Since the MHD time scale is much shorter than the time scale of slowing down of fast ion, AE analyses 
including the fast-ion distribution formation process demanded an exceedingly long computational time for the MEGA code. Therefore, the multi-phase MHD hybrid simulation, which is a combination of classical simulation and MHD hybrid simulation, has been developed[11-15]. The formation process of the steady state fast-ion distribution including the fast-ion redistribution brought about by the AEs can be simulated with the multi-phase simulation. The multi-phase simulation of the MEGA code was applied to the LHD experiment $\# 47645$ [8]. It was found that two groups of AEs with frequencies close to those observed in the experiment are destabilized alternately. The alternate appearance of multiple AEs is similar to the experimental observation[10].

On the other hand, the fast-ion losses due to the fast-ion driven instabilities in the LHD have not yet been investigated by using the MEGA code. In this work, we apply the multi-phase simulation to LHD plasmas with the temperature and the density profiles measured in LHD experiments and the realistic equilibrium magnetic field based on the temperature and the density profiles are used. In addition, a simple loss model in the divertor region is introduced to the MEGA code, and the fast-ion driven AEs and the fast-ion loss processes during the $\mathrm{AE}$ bursts are investigated.

The simulation model of the MEGA code is described in Sec. 2. In Sec. 3, a verification study of the MEGA code is conducted with the MORH code [16] on the fast-ion distribution in 
velocity space and fast-ion pressure profile. Fast-ion pressure profile has a significant effect on the AE stabilitiy. The MORH code has been used for the analyses of heating efficiency and fast-ion pressure in the LHD[16]. The fast-ion driven AEs and the fast-ion losses induced by the AEs in LHD are summarized in Sec. 4. The conclusion is presented in Sec. 5.

\section{Simulation Model of MEGA}

We use the MEGA code[17], in which the bulk plasma is described by the nonlinear MHD equations and the fast ions are simulated with the gyrokinetic particle-in-cell (PIC) method. Several hybrid simulation models have been constructed[17-23] to study the evolution of Alfven eigenmodes destabilized by fast ions. An extended MHD model given in Ref. 24 has been implemented together with the equilibrium toroidal flow in the MEGA code[25, 26]. In this paper, we use the standard MHD equations with the fast-ion effects

$$
\begin{aligned}
\frac{\partial \rho}{\partial t}= & -\nabla \cdot(\rho \mathbf{v})+v_{n} \Delta\left(\rho-\rho_{e q}\right), \\
\rho \frac{\partial}{\partial t} \mathbf{v}= & -\rho \mathbf{v} \cdot \nabla \mathbf{v}-\nabla p \\
& +\left(\mathbf{j}-\mathbf{j}_{h}^{\prime}\right) \times \mathbf{B}+\frac{4}{3} \nabla(v \rho \nabla \cdot \mathbf{v})-\nabla \times(v \rho \omega), \\
\frac{\partial p}{\partial t}= & -\nabla \cdot(p \mathbf{v})-(\gamma-1) p \nabla \cdot \mathbf{v} \\
& +(\gamma-1)\left[v \rho \omega^{2}+\frac{4}{3} v \rho(\nabla \cdot \mathbf{v})^{2}+\eta \mathbf{j} \cdot\left(\mathbf{j}-\mathbf{j}_{e q}\right)\right] \\
& +\chi \Delta\left(p-p_{e q}\right)
\end{aligned}
$$




$$
\begin{gathered}
\frac{\partial \mathbf{B}}{\partial t}=-\nabla \times \mathbf{E}, \mathbf{j}=\frac{1}{\mu_{0}} \nabla \times \mathbf{B} \\
\mathbf{E}=-\mathbf{v} \times \mathbf{B}+\eta\left(\mathbf{j}-\mathbf{j}_{e q}\right) \\
\omega=\nabla \times \mathbf{v}
\end{gathered}
$$

where $\mu_{0}$ is the vacuum magnetic permeability, $\gamma=5 / 3$ is the adiabatic constant, and $v, v_{n}$ and $\chi$ are artificial viscosity and diffusion coefficients chosen to maintain numerical stability. In this work, the dissipation coefficients $v, v_{n}, \chi$ and $\eta / \mu_{0}$ are assumed to be equal to each other. The dissipation terms play a physical role in enhancing the damping of AEs in the MHD simulation that does not include kinetic damping such as radiative damping[27] and thermal ion Landau damping. In this paper, we use one value of the coefficients, $2 \times 10^{-6}$, normalized by $V_{A} R_{0}$, where $V_{A}$ is the Alfvén velocity at the plasma center and $R_{0}$ is the major radius at the geometrical center of the simulation domain. The subscript "eq" represents the equilibrium variables. The MHD momentum equation [Eq. (2)] includes the fast-ion contribution in the fast-ion current density $\mathbf{j}_{h}^{\prime}$ that consists of the contributions from parallel velocity, magnetic curvature and gradient drifts, and magnetization current. The electromagnetic field is given by the standard MHD description. This model is accurate under the condition that the fast-ion density is much less than the bulk plasma density. The MHD equations are solved using a fourth order (in both space and time) finite difference scheme. The fast-ion current density is defined as follows. 


$$
\mathbf{j}_{h}^{\prime}=\mathbf{j}_{h \|}+\frac{1}{B}\left(P_{h \|} \nabla \times \boldsymbol{b}-P_{h \perp} \nabla \ln B \times \boldsymbol{b}\right)-\nabla \times\left(\frac{P_{h \perp}}{B} \boldsymbol{b}\right)
$$

where $\mathbf{j}_{h \|}$ denotes the parallel componet of the fast-ion current density. $P_{h \|}$ and $P_{h \perp}$ are parallel and perpendicular components of fast-ion pressure, respectively. These pressure components are calculated by using the full-f particle-in-cell (PIC) method based on a guiding-center approximation. The guiding center equations for each computational particle are solved using a fourth-order Runge-Kutta method and the linear interpolation. The $\mathbf{E} \times \mathbf{B}$ drift disappears in $\mathbf{j}_{h}^{\prime}$ due to quasi-neutrality[17].

We would like to investigate the fast-ion distribution formation process with beam injection, collisions, losses, and transport due to the AEs. A complicating factor is that the time scale of the classical processes without MHD perturbations is the slowing-down time, which is roughly $100 \mathrm{~ms}$, and longer by four orders of magnitude than the typical oscillation period of AEs $\sim 0.01$ ms. The time step width is limited by the Courant condition for fast magnetosonic waves in the hybrid simulation. On the other hand, in the classical simulation, where the MHD part of the simulation is turned off, the time step width can be taken to be greater by one order of magnitude than in the hybrid simulation. To deal with this efficiently, a multi-phase simulation, where the classical simulation and the hybrid simulation are run alternately, was constructed[11-15]. In the classical phase of the simulation, the fast-ion distribution is built up with the beam injection and collisions. In the subsequent hybrid phase, the built-up fast-ion 
distribution destabilizes AEs leading to the relaxation of the distribution. We should note that the classical process, beam injection and collisions, also take place in the hybrid phase. We repeat this combination of the classical and hybrid simulations until the stored fast-ion energy is saturated.

\section{Verification on fast-ion velocity distribution and pressure profile}

As a preparatory step for applying the MEGA code to the AE analyses of the LHD experiments, a verification study is conducted between the MEGA code and the MORH code on fast-ion distribution in velocity space and fast-ion pressure profiles that are produced by the neutral beam (NB) and collisions. Fast-ion pressure profile has a large effect on the fast-ion driven instabilities. The MORH code is a drift kinetic orbit-following Monte Carlo code using the HINT[28, 29] equilibrium[16]. In the MORH code, the $6^{\text {th }}$ order Runge-Kutta method[30] and the $4^{\text {th }}$ order spline interpolation method[31] are used for solving the guiding center equation. The heating efficiency and the pressure of fast ions produced by NBs have been calculated for the LHD plasma reported in Ref. 8.

Figure 1 compares the MEGA and the MORH results for fast-ion velocity distribution. The fast-ion pressure profiles are compared in Fig. 2. In these figures, calculation conditions such as the temperature and density profiles and the magnetic field strength are the same as the TAE 
discharge in the LHD which is reported in Ref. 8. In this case, three tangential-NB are injected.

Thus, the parallel component of fast-ion pressure is higher than the perpendicular component as we see in Fig. 2. In these figures, good agreements are found between MEGA and MORH for both the fast-ion velocity distribution and the pressure profiles.
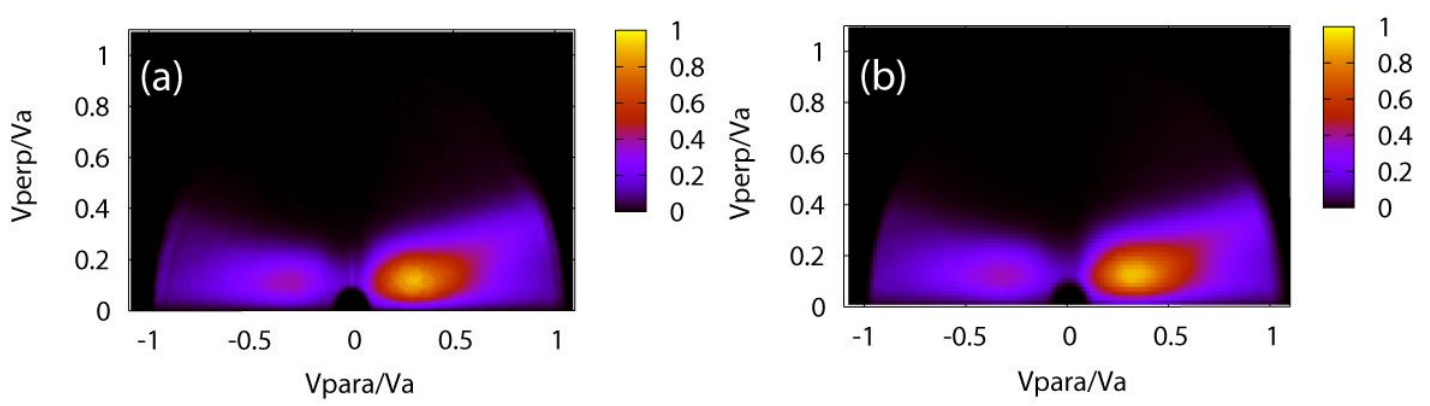

Fig. 1 Fast-ion velocity distribution calculated with (a) MEGA and (b) MORH. Horizontal axes and vertical axes are parallel and perpendicular fast-ion velocities normalized by the Alfvén velocity, respectively. The color represents the number of fast ions [arb.unit].
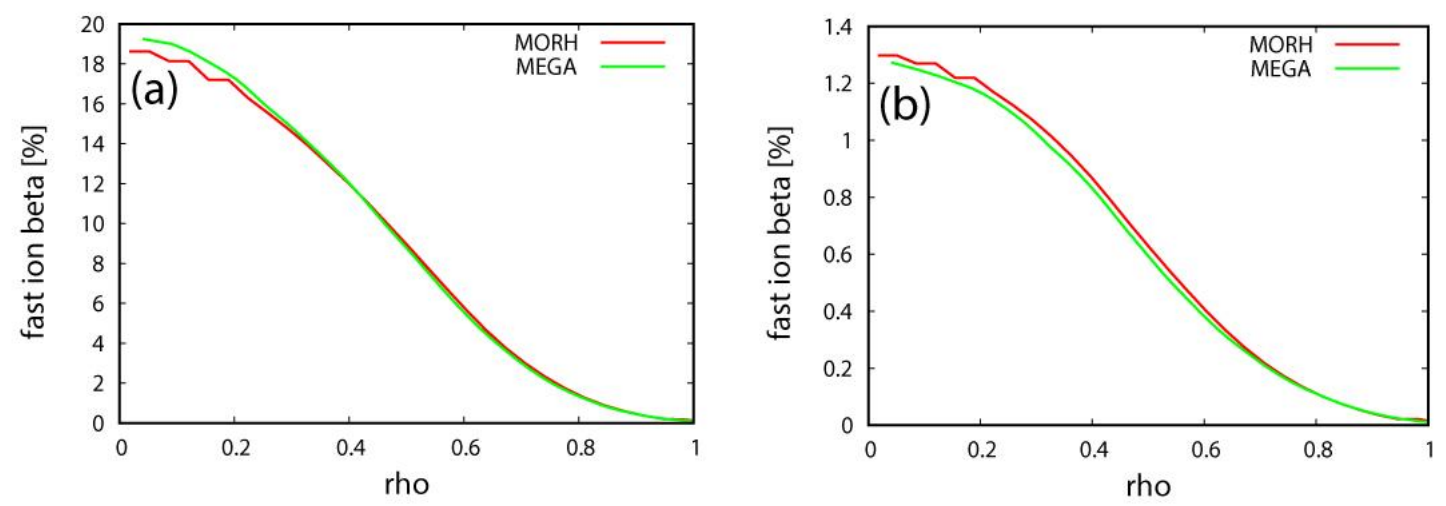
Fig. 2 Comparison of MEGA and MORH for (a) parallel fast-ion beta profile and (b)

perpendicular fast-ion beta profile. Horizontal axis is the normalized minor radius. Green and

red lines represent the results of MEGA code and MORH code, respectively.

\section{Fast-ion driven AEs and fast-ion losses induced by AEs in LHD}

\subsection{Simulation condition}

In order to investigate the fast-ion loss process due to the TAE burst, a multi-phase simulation of MEGA code is applied to the LHD experiment \#90090[9], in which the fast-ion losses were observed during the TAE burst. Because the interval of TAE burst in the LHD experiment is about $5 \mathrm{~ms}$, the classical simulation and the hybrid simulation are alternately run for $4 \mathrm{~ms}$ and 1 ms, respectively. The equilibrium magnetic field is calculated by the HINT2 code[28, 29] based on the profiles of electron density and temperature measured in the LHD experiment. The field strength at the magnetic axis is 0.6T. In this experiment, three tangential-injected NBs composed of the two co-injected NBs and one counter-injected NB are used. In this paper, the "co-" means the direction of the toroidal current increasing the poloidal field. The "counter-" denotes the opposite direction. In the LHD, the "co-" direction approximately corresponds to the direction of the toroidal magnetic field. Pitch angle is the angle between the particle velocity vector and the magnetic field. The pitch angle $\theta$ is defined by $\cos (\theta)=\mathrm{v}_{\|} / \mathrm{v}$, where $\mathrm{v}_{\|}$and $\mathrm{v}$ are 
parallel and total velocity, respectively. Thus, the pitch angle of co-injected fast-ion is $\sim 0$ while the pitch angle of the counter-injected fast-ion is $\sim \pi$. The injection power of each NB is about 5 MW. Thus, the injection powers of co-injected NB and counter-injected NB are about 10 MW and 5 MW, respectively. Using the density and temperature profiles shown in Fig. 3, the birth profile of fast ions, which is used as the fast-ion source of the MEGA code, are calculated by the HFREYA code with considering the injection energies of each NB. The total absorption power is about 9 MW. Figure 4 shows the fast-ion birth profile produced by the three NBs.

In this multi-phase simulation, 100,000 simulation particles per $1 \mathrm{~ms}$ are added in order to calculate the time evolution of fast ion produced by NB. Some million simulation particles are used when stored energy of fast ion is saturated $(\sim 50 \mathrm{~ms})$. The numbers of grid points are $(128$, $640,128)$ for cylindrical coordinates $(\mathrm{R}, \varphi, \mathrm{z})$. The simulations of the AE bursts were run with 8 、 192 cores/256 nodes of "Plasma Simulator", which is a large scale parallel supercomputer, for about 100 hours.

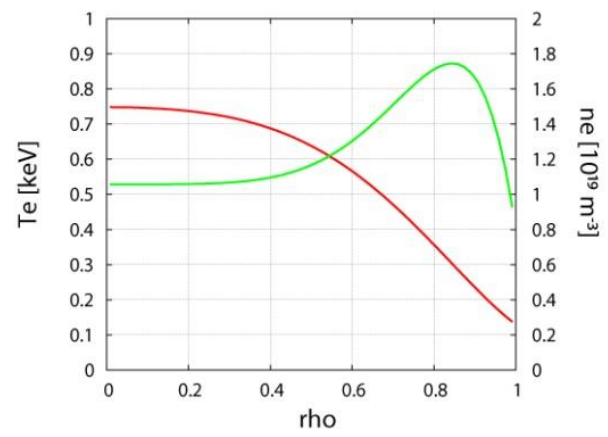

Fig. 3 Electron temperature and density profiles in LHD experiment \#90090. 


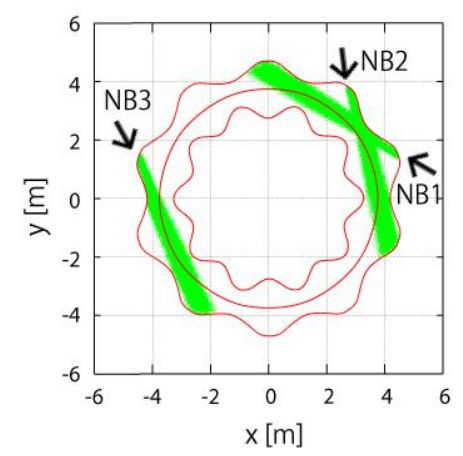

Fig. 4 Fast-ion birth profile produced by NB. Green points represent the birth points projected onto the equatorial plane. Red lines represent the last closed flux surface (LCFS) and the magnetic axis.

\subsection{Fast-ion driven AEs and the fast-ion losses}

\subsubsection{Time evolution of AEs and fast-ion losses}

The time evolutions of MHD kinetic energy of fast-ion driven instabilities, fast-ion loss rate due to the instabilities, and stored fast-ion energy in the multi-phase simulation with MEGA code are shown in Fig. 5. In Fig. 5(b) and (c), the results of the "classical calculation" which are results of MEGA code without MHD instabilities, are shown for comparison. In Fig. 5(b), fast-ion loss rate does not include the promptly lost particles whose lifetime is less than $50 \mu \mathrm{s}$. We see in Fig. 5(a) that the recurrent bursts of fast-ion driven instabilities occur. The fast-ion loss rate shown in Fig. 5(b) significantly increases associated with each burst of the instabilities. In addition, the fast-ion loss rate in the classical phase of the multi-phase simulation becomes 
larger than the "classical calculation." This can be attributed to the fast-ion redistribution due to the instabilities during the hybrid phase of the multi-phase simulation. We see in Fig. 5(c) that the increase of fast-ion loss results in the lower saturation levels of stored fast-ion energy than the "classical calculation." It is noted that the confinement of counter-injected fast-ion is much lower than that of co-injected fast-ion in the case of $0.6 \mathrm{~T}$ [32].
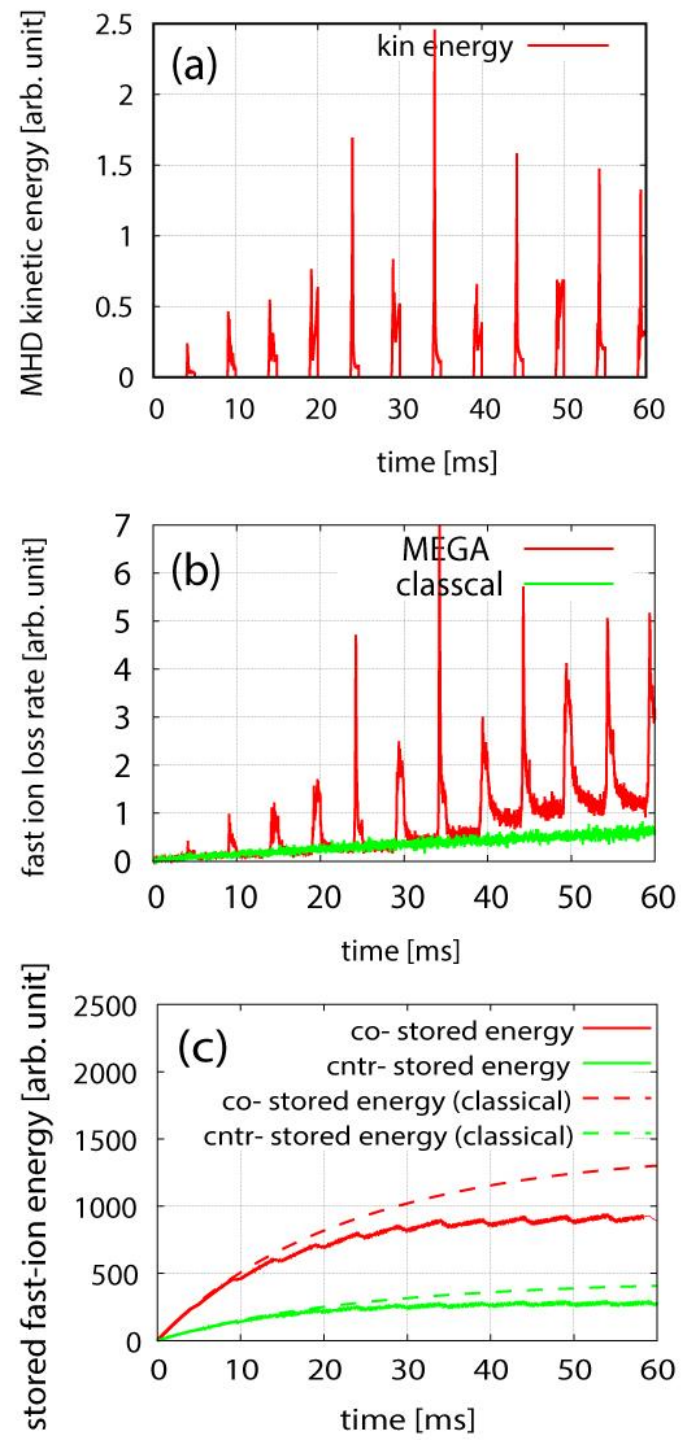
Fig. 5 Time evolutions of (a) MHD kinetic energy of the fast-ion driven instabilities, (b) fast-ion loss rate, and (c) stored fast-ion energy. In panels (b) and (c), the results of the "classical calculation," which are results of MEGA code without MHD instabilities, are shown together for comparison. In panel (b), fast-ion loss rate does not include the prompt loss particles whose lifetime is less than $50 \mu \mathrm{s}$.

\subsubsection{Typical AE burst after the saturation of stored fast-ion energy}

We discuss the relationship between the fast-ion loss and the radial MHD velocity harmonics of the fast-ion driven instabilities during a typical burst. Figure 6 shows the time evolution of the radial MHD velocity harmonics during a burst of fast-ion driven instabilities after the saturation of stored fast-ion energy $(\mathrm{t}=54 \mathrm{~ms}-55 \mathrm{~ms})$. We see in Fig. $6(\mathrm{a})$ that the burst consists of three peaks. Figure $6(\mathrm{~b})$ shows the time evolution of the primary harmonic for each peak. The primary mode number at the initial peak is $\mathrm{m} / \mathrm{n}=1 / 1$ ( $\mathrm{m}$ is a poloidal mode number and $\mathrm{n}$ is a toroidal mode number). And then, the instability with $\mathrm{m} / \mathrm{n}=2 / 1$ becomes large. At the maximum level of MHD kinetic energy during the burst, the primary harmonic of radial MHD velocity is $\mathrm{m} / \mathrm{n}=2 / 1$. It is found in Fig. $6(\mathrm{~b})$ and (c) that the fast-ion loss rate takes the maximum value near the peak of the $m / n=2 / 1$ mode amplitude. 

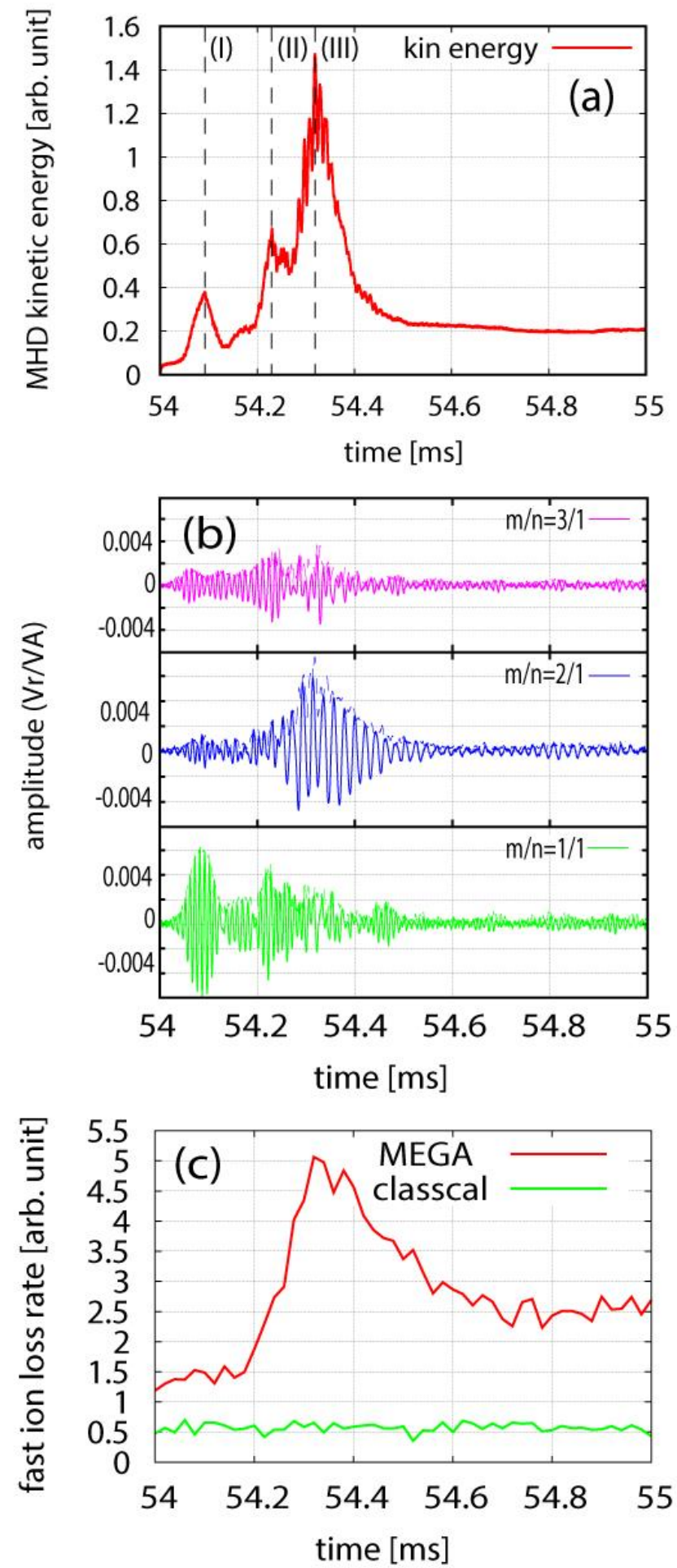

Fig. 6 Time evolutions of (a) MHD kinetic energy, (b) radial MHD velocity harmonics, and (c) fast-ion loss rate during a burst of fast-ion driven instabilities. 
Next, the frequencies of the harmonics are investigated. Figure 7(a) shows the frequency spectra of radial MHD velocity harmonics with $m / n=1 / 1, m / n=2 / 1$ and $m / n=3 / 1$. It is found in Fig. 7 (a) that the frequency of the $\mathrm{m} / \mathrm{n}=1 / 1$ harmonic, which initially appears in the burst, is about $100 \mathrm{kHz}$. The frequency of the $\mathrm{m} / \mathrm{n}=2 / 1$ harmonic which is dominant at the peak of the MHD kinetic energy is $48 \mathrm{kHz}$. The frequency of the $\mathrm{m} / \mathrm{n}=3 / 1$ harmonic is $80 \mathrm{kHz}$. The radial profiles of the radial MHD velocity harmonics with frequencies $48 \mathrm{kHz}, 80 \mathrm{kHz}$ and $100 \mathrm{kHz}$ are shown in Fig. 7(b), (c), and (d), respectively.

The frequency and the location are shown for each mode with the Alfvén continuous spectra for the major toroidal harmonics $n=1$ and $n=11$ in Fig. 8. The Alfvén continuous spectra were analyzed with the STELLGAP code [33]. The mode with frequency $48 \mathrm{kHz}$ has the dominant harmonics with $\mathrm{m} / \mathrm{n}=2 / 1$ that peaks around $\mathrm{r} / \mathrm{a}=0.4$. The peak shifts inward from the TAE gap at rho $=0.6$, but the frequency is located well inside the spectrum gap. The mode might be a TAE with the profile affected by fast-ions. The mode with frequency $80 \mathrm{kHz}$ has two dominant harmonics with $\mathrm{m} / \mathrm{n}=1 / 1$ and $\mathrm{m} / \mathrm{n}=3 / 1$. Since the frequency is almost coincident with ellipticity-induced gap shown in Fig. 8 , the mode with $\mathrm{m} / \mathrm{n}=1 / 1$ and $\mathrm{m} / \mathrm{n}=3 / 1$ is classified as an ellipticity-induced Alfvén eigenmode (EAE). The mode with frequency $100 \mathrm{kHz}$ has the 
dominant harmonic with $\mathrm{m} / \mathrm{n}=1 / 1$ that peaks near the plasma center. The frequency is close to the gap near the plasma center. The mode may be a global Alfvén eigenmode (GAE).

Next, we discuss the effect of the AEs on the fast-ion pressure profile. The parallel fast-ion beta profiles during the burst are shown in Fig. 9. In Fig. 9, the fast-ion beta profiles are shown for 4 moments: (I) the initial peak of the MHD kinetic energy, (II) the second peak, (III) the maximum peak, and before burst. The moments (I), (II) and (III) are indicated by dashed lines in Fig. 6(a). At the moment (I), the fast-ion beta slightly decreases only near the plasma center. At the moments (II) and (III), the fast-ion beta near the plasma center region significantly decreases while the fast-ion beta becomes larger near the peripheral region than that before the burst. This result indicates that the fast-ions are transported from the plasma center region to the peripheral region due to the $\mathrm{AE}$ instabilities. This radial transport of fast ions brings about the larger fast-ion loss rate than the classical calculation after the burst as described in section 4.2.1. 

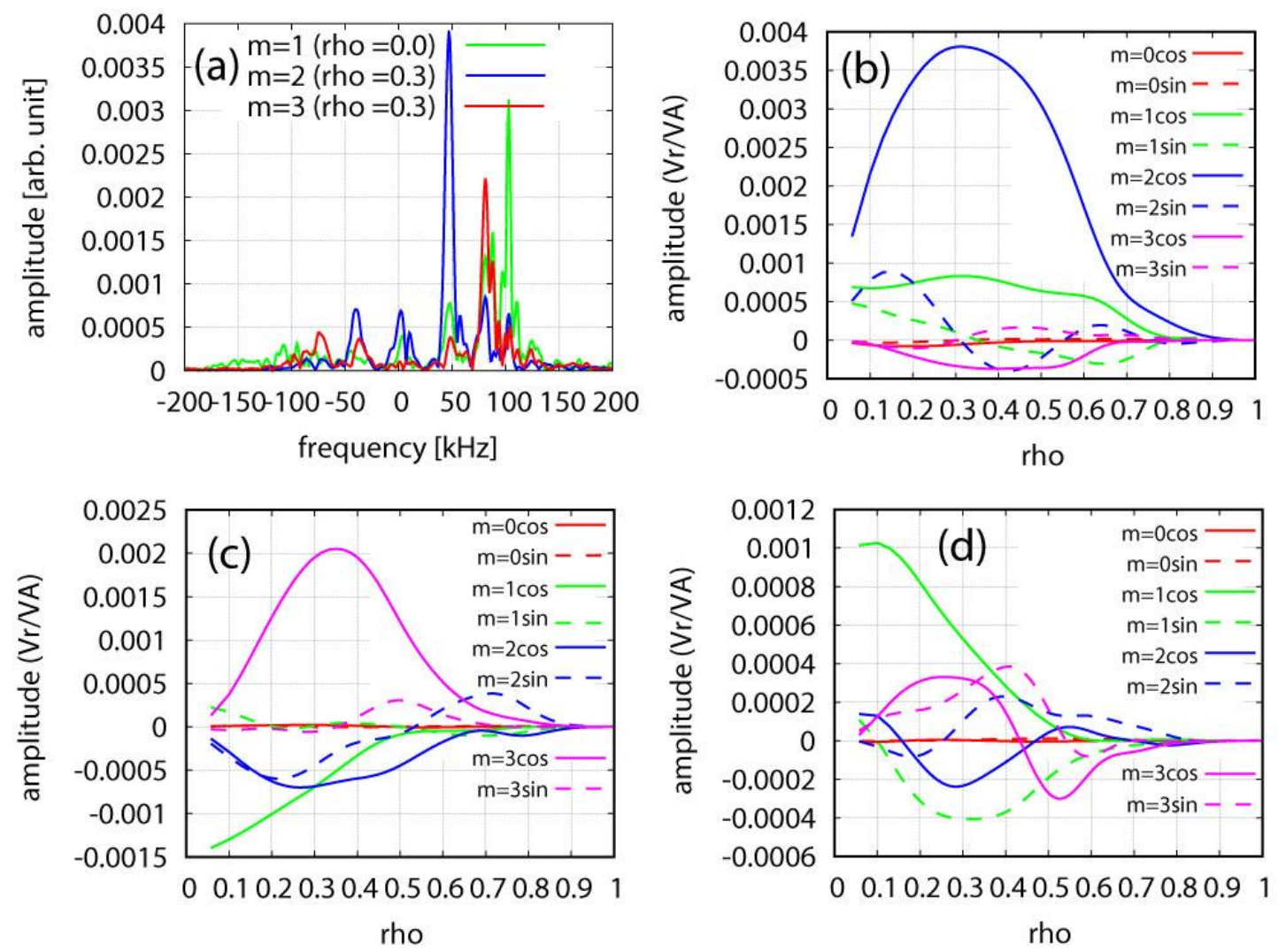

Fig. 7 Frequency spectra of radial MHD velocity harmonics with $m / n=1 / 1,2 / 1$, and 3/1; (a) and radial profiles of the modes for the peaks in the frequency spectra, (b) $48 \mathrm{kHz}$, (c) $80 \mathrm{kHz}$, and (d) $100 \mathrm{kHz}$.

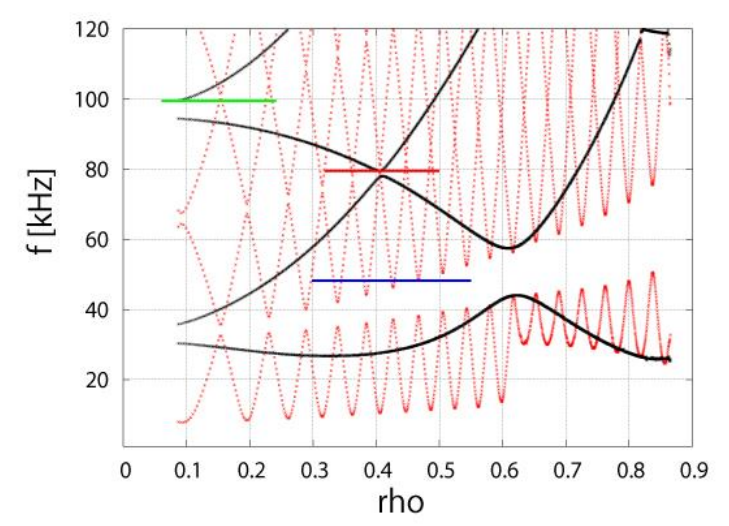


Fig. 8 Alfvén continuous spectra of the toroidal mode numbers $n=1$ (black) and $n=11$ (red). The frequency and the spatial location for each $\mathrm{AE}$ with frequency $\mathrm{f}=48,80,100 \mathrm{kHz}$ is shown with horizontal line.

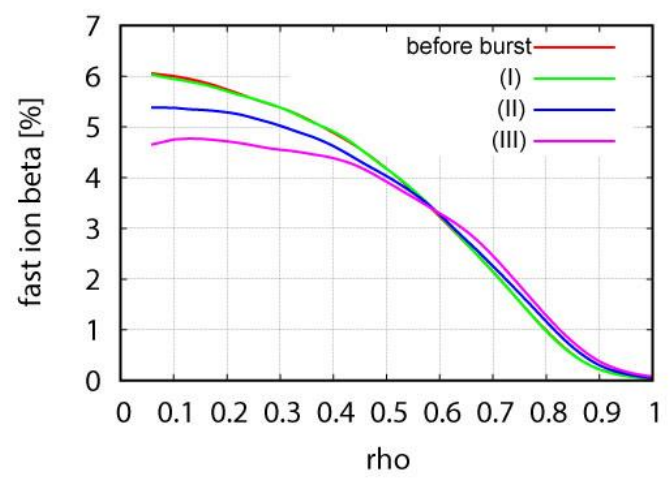

Fig. 9 Fast-ion beta profiles during the burst of the fast-ion driven instabilities. Red, green, blue and purple lines represent the fast-ion beta profiles at 4 moments: (I) the initial peak of MHD kinetic energy, (II) the second peak, (III) the maximum peak, and before burst. The moments (I), (II), and (III) are indicated by dashed lines in Fig. 6 (a).

\subsection{Properties of fast-ion loss induced by AEs}

The effect of AE amplitude on fast-ion loss rate is investigated. Figure 10 shows the AE-induced fast-ion loss rate versus the maximum AE amplitude for each burst. We see that the fast-ion loss rate brought about by the $\mathrm{AE}$ burst is proportional to the square of $\mathrm{AE}$ amplitude. This quadratic dependence of fast-ion loss was measured in the LHD experiments [9]. 


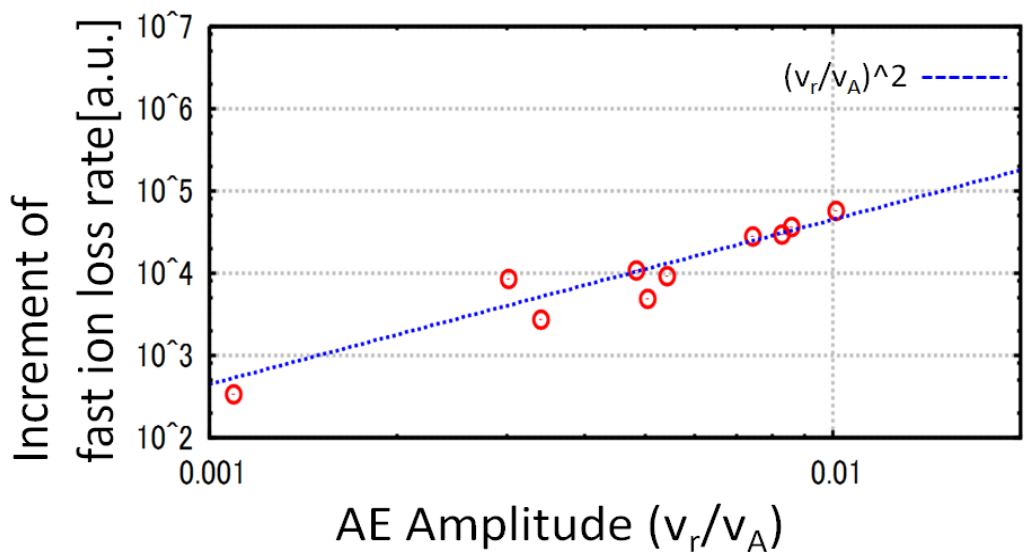

Fig. 10 AE-induced fast-ion loss rate versus maximum AE amplitude (radial MHD velocity normalized by Alfvén velocity) for each burst.

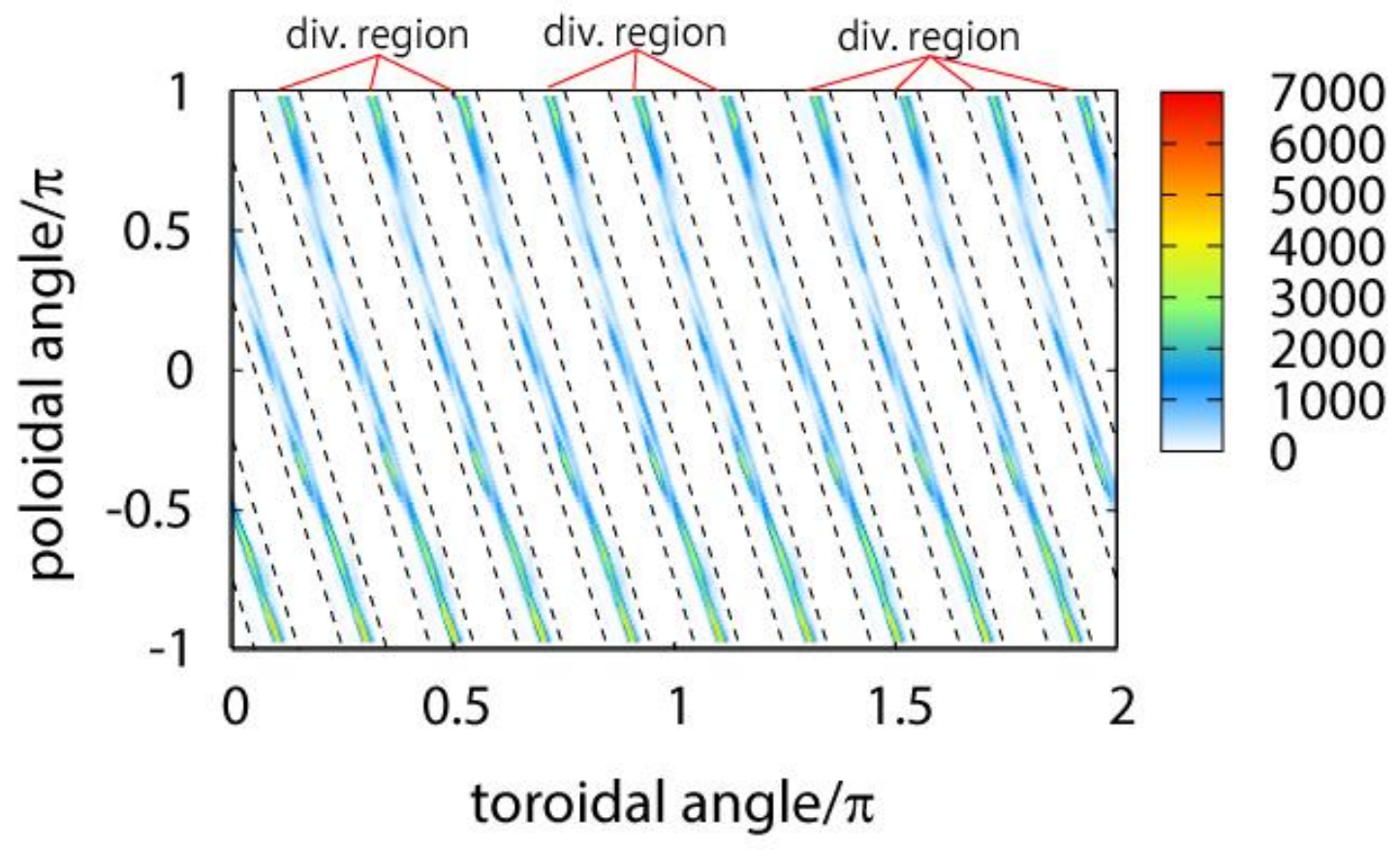

Fig. 11 Distribution of lost fast ions. Color represents the number of fast ions [arb.unit]. Black dashed lines show the divertor regions. 
We investigate in detail the properties of fast-ion loss induced by the AEs. Figure 11 shows the distribution of lost fast ions. We see in Fig. 11 that almost all the lost fast ions are located in the divertor regions. In the LHD, most of the lost fast ions can move to diverter regions along the field lines because the diverter regions are the regions of weaker magnetic field strength. We have examined the time evolution of the velocity distribution of lost fast-ions in the divertor region. In Fig. 12, the time evolution of the fast-ion loss rate is shown for those produced by the co-injected NB and the counter-injected NB. The main component of the lost fast-ions is those produced by the counter-injected NB. On the other hand, the lost fast-ions produced by co-injected NB slightly increase during the AE burst and slowly decrease after the AE burst.

Next, we discuss the velocity distribution of the lost fast-ions. Figures 13 and 14 compare the time evolution of total velocity and pitch-angle distribution, respectively, between the multi-phase simulation and the classical simulation. In the classical simulation shown in Fig. 13(b), the fast-ion losses occur near the beam injection velocity. The particles are so-called prompt loss particles. A few of the fast ions near the thermal velocity are lost due to the Coulomb collision with the bulk plasma. In Fig. 13(a), the loss of fast ions near the injection velocity becomes much larger during the $\mathrm{AE}$ burst. In addition, the fast-ion losses increase also near the thermal velocity. 
Next, the pitch angle of lost fast ions is investigated. In the classical simulation shown in Fig. 14(b), there are two peaks near pitch angle $\sim 0.75 \pi$ and $\sim 0.9 \pi$. The pitch angle $\sim 0.9 \pi$ is almost the same as the initial pitch angle of the counter-injected NB. The fast ions with pitch angle $~$ $0.75 \pi$ correspond to "transit particles" which transit between particles trapped in a helical ripple and particles trapped in a toroidal ripple. The "transit particle" is called "chaotic-orbit particle" in reference [34]. The pitch angel of $0.75 \pi$ is almost the same as the pitch angle measured by the scintillator-based lost fast-ion probe [9] when we consider the reflection of the trapped particle due to the mirror force. Since the "transit particle" does not form close orbit surface, the "transit particle" tends to be quickly lost. Compared between Fig. 14(a) and Fig. 14(b), many of the fast ions with pitch angle $\sim 0.9 \pi$ are lost during the $\mathrm{AE}$ burst. In the peak at the pitch angle $\sim 0.75 \pi$, the number of lost fast ions is kept larger after AE burst as well as during the AE burst. The loss of the fast ions with pitch angle $\sim 0.1 \pi$ is slightly larger than the "classical simulation." 


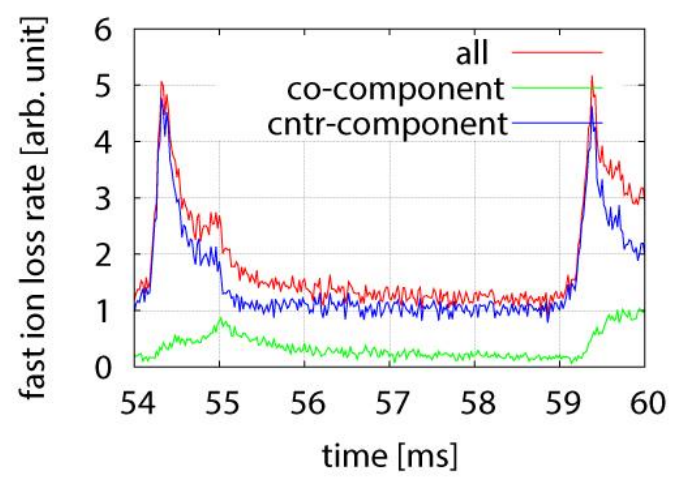

Fig. 12 Time evolution of fast-ion loss rate. Green and blue lines represent the fast-ion loss rate for the fast ions produced by the co- and the counter-injected NBs, respectively. Red line represents the total fast-ion loss rate. The hybrid phase of the multi-phase simulation is $54 \mathrm{~ms}-55 \mathrm{~ms}$ and $59 \mathrm{~ms}-60 \mathrm{~ms}$, and the classical phase where the MHD perturbations are neglected is $55 \mathrm{~ms}-59 \mathrm{~ms}$.
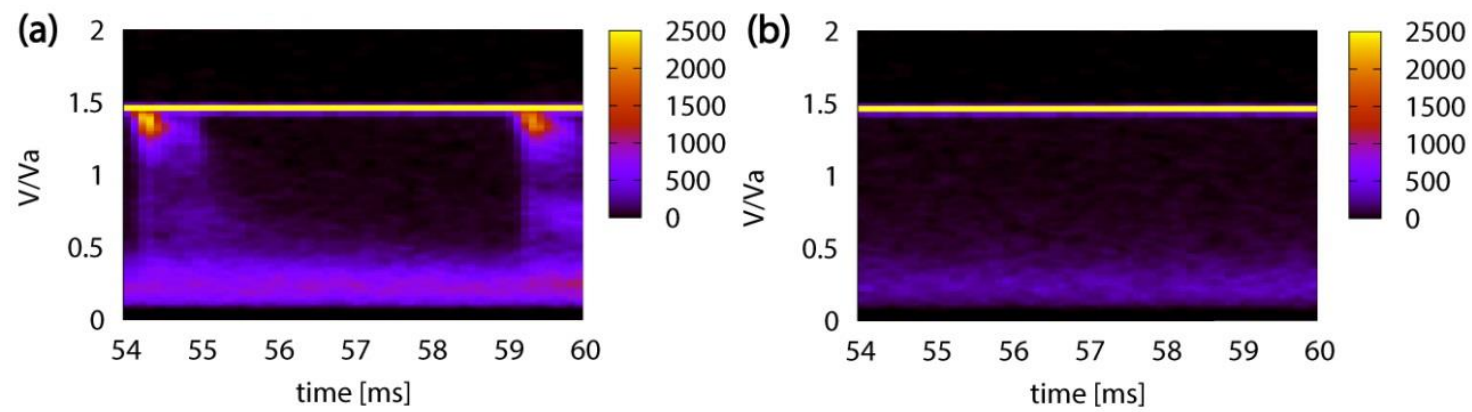

Fig.13 Time evolution of total velocity distribution of lost fast ions for (a) multi-phase and (b)

classical simulations. Color represents the number of fast ions [arb.unit]. The hybrid phase of the multi-phase simulation is $54 \mathrm{~ms}-55 \mathrm{~ms}$ and $59 \mathrm{~ms}-60 \mathrm{~ms}$, and the classical phase where the MHD perturbations are neglected is $55 \mathrm{~ms}-59 \mathrm{~ms}$ in Fig. (a). 

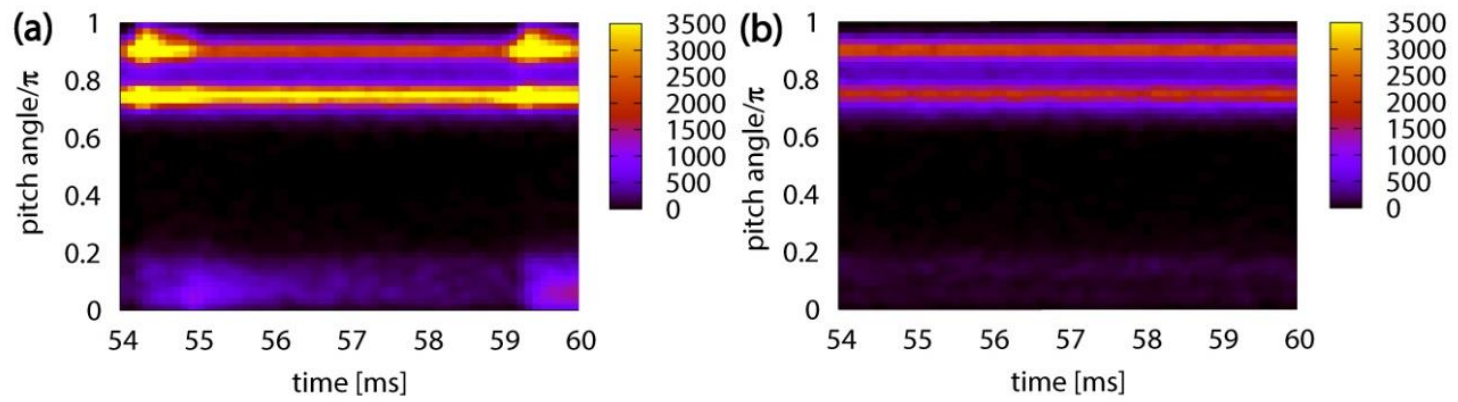

Fig.14 Time evolution of pitch-angle distribution of lost fast ions for (a) multi-phase and (b)

classical simulations. Color represents the number of fast ions [arb.unit]. The hybrid phase of the multi-phase simulation is $54 \mathrm{~ms}-55 \mathrm{~ms}$ and $59 \mathrm{~ms}-60 \mathrm{~ms}$, and the classical phase where the MHD perturbations are neglected is $55 \mathrm{~ms}-59 \mathrm{~ms}$.

In order to understand the difference of fast-ion loss in the two peaks of pitch angle, the time evolution of the pitch angle distribution from the start time to the end of calculation is shown in Fig. 15. In the classical simulation shown in Fig. 15(b), the fast-ion loss rate with pitch angle $\sim 0.9 \pi$ is kept constant while the lost fast ions with pitch angle $\sim 0.75 \pi$ increase along with the time development. The slowing down time at the center region $(\mathrm{rho}=0.1)$ and near the peripheral region (rho=0.9) is $35 \mathrm{~ms}$ and $3.7 \mathrm{~ms}$, respectively. The lost fast ions with pitch angle $\sim 0.75 \pi$ increase after slowing down. Compared between Fig. 15(a) and Fig. 15(b) in the earlier time, there is not any difference in the lost fast ions with pitch angle $\sim 0.75 \pi$ except for the hybrid phase. After the saturation of stored fast-ion energy (50ms-60ms), the number of lost fast ions with pitch angle $\sim 0.75 \pi$ in Fig. 15(a) is larger than that in Fig. 15(b) as discussed for Fig. 
14. The difference for pitch angle $\sim 0.75 \pi$ is caused by the fast ions transported due to the $\mathrm{AE}$ burst. The fast ions transported near the peripheral region slow down in a shorter time. Therefore, the number of "transit particles" becomes large because of the strong pitch angle scattering after slowing down.

In order to clarify a relationship between velocity and pitch angle of lost fast ion, the number of lost fast ions is plotted in pitch angle and total velocity space in Fig. 16. The lost fast ions with pitch angle $\sim 0.9 \pi$ are found to have the total velocity near the initial velocity. On the other hand, the lost fast ions with pitch angle $\sim 0.75 \pi$ are near the thermal velocity. These lost fast ions with pitch angle $\sim 0.75 \pi$ become "transit particles" because the pitch angle scatters after slowing down. In the low magnetic field strength, most of "transit fast ions" are quickly lost than the collision time because the "transit particle" does not form close orbit surface. 

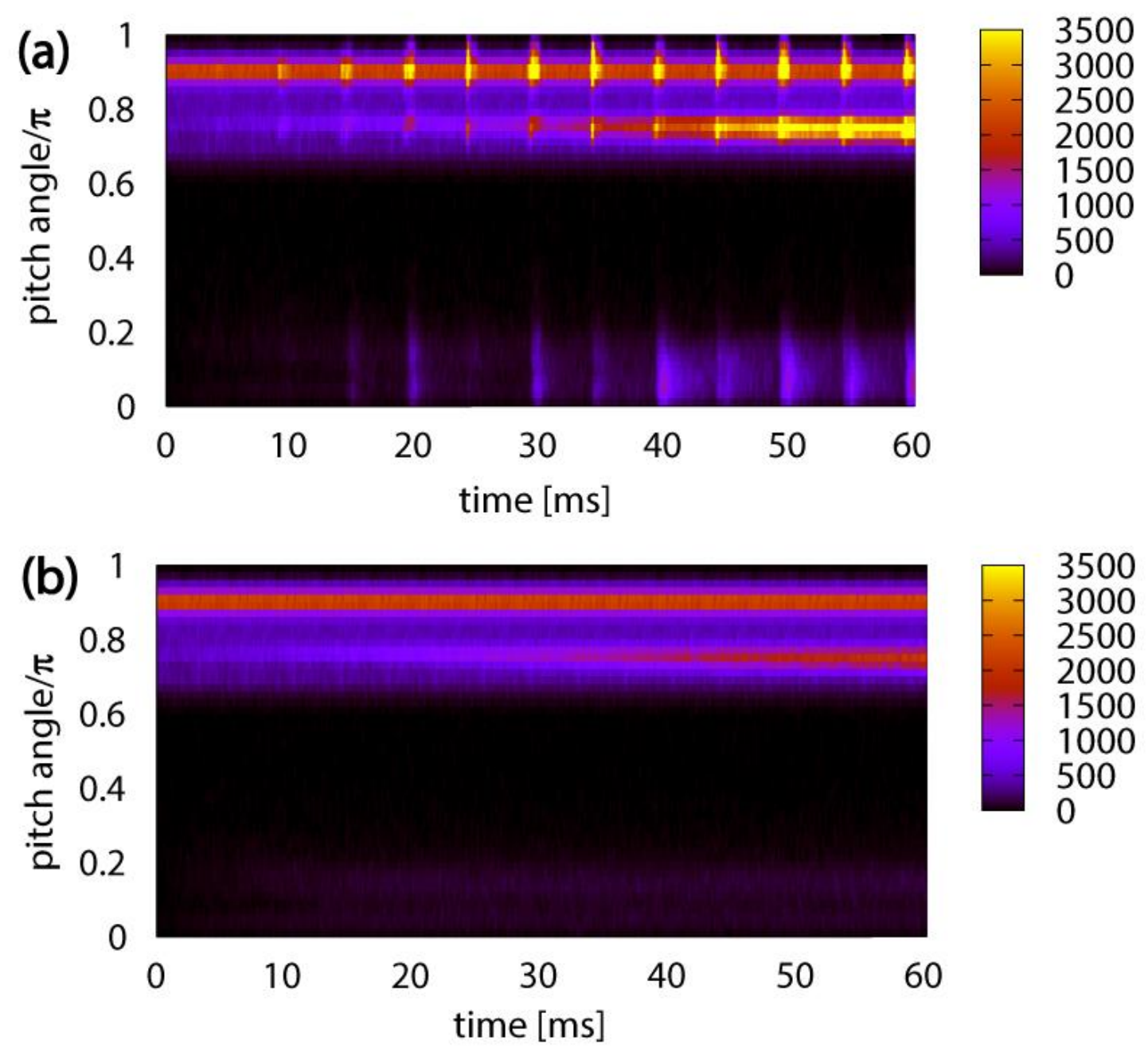

Fig.15 Time evolution of pitch-angle distribution of lost fast ions for (a) multi-phase and (b)

classical simulations from the start time to the end of calculation. Color represents the number of fast ions [arb.unit]. Fast-ion loss rate is large during the hybrid phase of the multi-phase simulation in panel (a). 


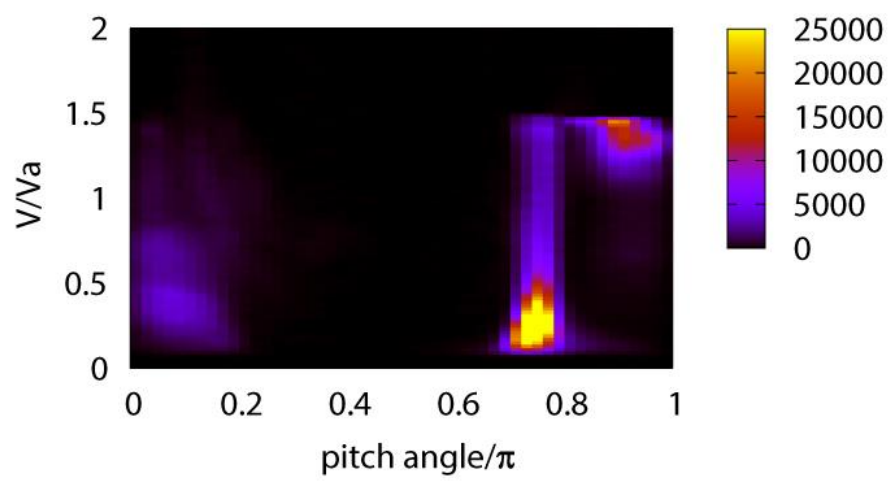

Fig. 16 Velocity space distribution of lost fast ions in the multi-phase simulation. Horizontal axis is pitch angle normalized by $\pi$, and the vertical axis is the total velocity normalized by Alfvén velocity. Color represents the number of fast ions [arb. unit].

Next, we investigate the initial distribution of the lost fast ions due to the AEs. Figure 17 shows the time evolution of the initial pitch angle distribution of the lost fast ions. The time evolution of the initial radial distribution of the lost fast ions produced by the co-injected NB and the counter-injected NB are shown in Figs. 18 and 19, respectively. It is found in Fig. 17 (a) that the number of lost fast ions with initial pitch angle $\sim 0.9 \pi$ becomes larger during the $\mathrm{AE}$ burst. This peak is the same as the pitch angle of the lost fast ions shown in Fig. 14 (a). This result indicates that the variation in the pitch angle of the lost fast ions is small during the $\mathrm{AE}$ burst.

The fast-ion loss with the initial pitch angle $\sim 0.1 \pi$, which indicates that these particles are produced by co-injected NB, clearly increases after the AE burst as well as during the AE burst. 
Most of these particles produced by co-injected NB can slow down to near the thermal velocity because their orbits are not easy to be lost [32]. After slowing down, these particles become "transit particles." As a result, their pitch angle is near $0.75 \pi$ when they are lost. In other words, the AE-induced loss of fast ions produced by co-injected NB is caused by the conversion to transit particles with the pitch angle $\sim 0.75 \pi$, which is shown in Fig 14(a).

Next, the time evolution of the initial radial distribution of the lost fast ions is shown in Figs. 18 and 19 for the co-injected NB and the counter-injected NB, respectively. We see in Fig. 18(a) that fast ions produced in the peripheral region (rho> 0.9) by the co-injected NB are mainly lost when the fast-ion driven AEs appear. For the fast ions produced by the counter-injected NB shown in Fig. 19(a), the loss of the fast ions with initial position rho $>0.5$ becomes large in the early phase of the AE burst. And then, the fast ions produced near the plasma center region (rho $\sim 0.0$ ) are lost near the maximum amplitude of the instabilities.
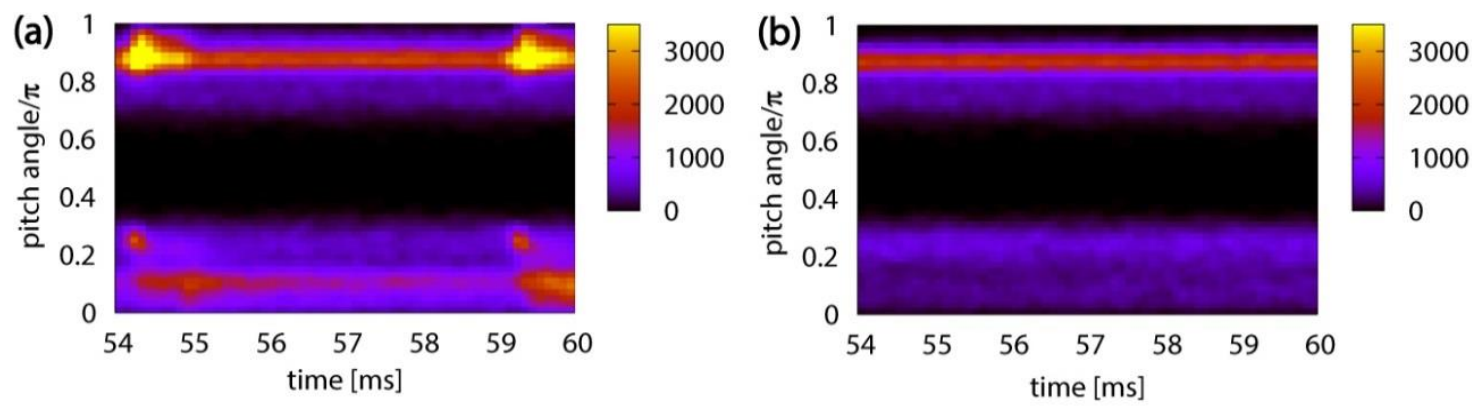
Fig. 17 Time evolution of the initial pitch angle distribution of lost fast ions for (a) multi-phase and (b) classical simulations. Color represents the number of lost fast ions [arb.unit]. The hybrid phase of the multi-phase simulation is $54 \mathrm{~ms}-55 \mathrm{~ms}$ and $59 \mathrm{~ms}-60 \mathrm{~ms}$, and the classical phase where the MHD perturbations are neglected is $55 \mathrm{~ms}-59 \mathrm{~ms}$.
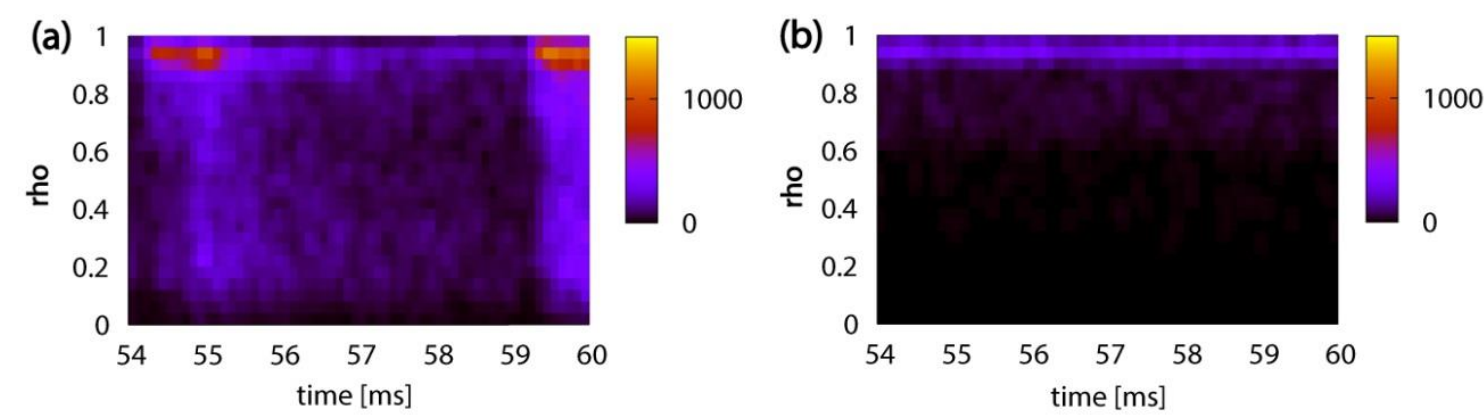

Fig. 18 Time evolution of the initial radial distribution of lost fast ions produced by co-injected NB for (a) multi-phase and (b) classical simulations. The initial radial distribution is the distribution of the birth radial positions of the lost fast ions when they are ionized from the neutral particles. Color represents the number of fast ions [arb.unit]. The hybrid phase of the multi-phase simulation is $54 \mathrm{~ms}-55 \mathrm{~ms}$ and $59 \mathrm{~ms}-60 \mathrm{~ms}$, and the classical phase where the MHD perturbations are neglected is $55 \mathrm{~ms}-59 \mathrm{~ms}$. 

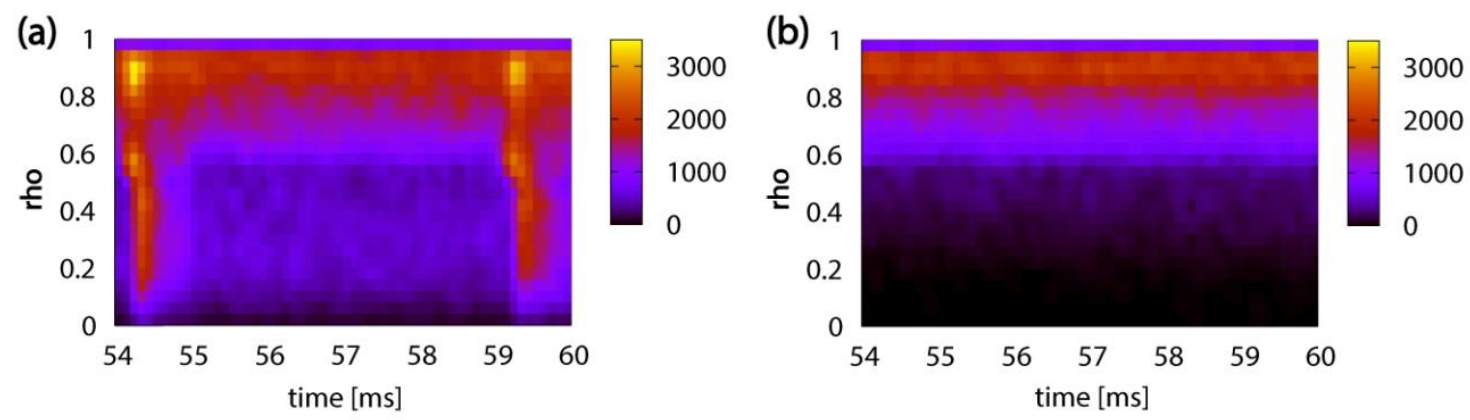

Fig. 19 Time evolution of the initial radial distribution of lost fast ions produced by counter-injected NB for (a) multi-phase and (b) classical simulations. The initial radial distribution is the distribution of the birth radial positions of the lost fast ions when they are ionized from the neutral particles. Color represents the number of fast ions [arb.unit]. The hybrid phase of the multi-phase simulation is $54 \mathrm{~ms}-55 \mathrm{~ms}$ and $59 \mathrm{~ms}-60 \mathrm{~ms}$, and the classical phase where the MHD perturbations are neglected is $55 \mathrm{~ms}-59 \mathrm{~ms}$.

\section{Conclusion}

We applied the MEGA code to LHD plasmas with the temperature and density profiles measured in LHD experiments and the realistic equilibrium magnetic field based on the temperature and density profiles. A verification study of the MEGA code was conducted with the MORH code on the classical fast-ion distribution in velocity space and the classical fast-ion pressure profile. Good agreements were found between MEGA and MORH on the classical distribution and pressure profile where the MHD perturbations are not considered. The multi-phase simulations were performed with the MEGA code for the LHD experiment where 
fast-ion losses were observed associated with the AE bursts. The fast-ion driven AE instabilities and fast-ion loss induced by AEs are investigated.

In the multi-phase simulation for the LHD experiment, the AE bursts with the dominant mode $\mathrm{m} / \mathrm{n}=2 / 1$ appears recurrently. The fast-ion loss significantly increases associated with each burst of the instabilities. As a result of their loss, the stored fast-ion energy is saturated at a lower level than that of the "classical calculation."

The main component of the lost fast ions induced by the $\mathrm{AE}$ burst are the fast ions produced by counter-injected NB. The increment of the fast-ion loss rate is proportional to the square of AE amplitude. This quadratic dependence of fast-ion loss rate was observed in the LHD experiment. In addition, the fast-ion losses are larger than the classical calculation even after the bursts. These lost fast-ions have velocity near the thermal velocity and pitch angle which corresponds to "transit particles."

We investigated the initial location of the lost fast ions. For the counter-injected fast-ions, the fast-ions deposited well inside the plasma up to the magnetic axis are significantly lost during the AE burst. The majority of the lost fast ions produced by co-injected NB are initially located in the peripheral region.

The velocity space region of lost fast ions was measured during TAE bursts by scintillator-based lost fast-ion probe in the LHD experiment \#90090[9]. We will compare the 
velocity distribution of lost fast ions in the MHD hybrid simulation with the experiment. In addition, the properties of the fast-ion transport due to the AE bursts in the LHD will be examined in the near future.

Acknowledgments

Numerical computations were performed at the Plasma Simulator (FUJITSU FX100) of NIFS with the support and under the auspices of the NIFS Collaboration Research programs (NIFS16KNSR005, NIFS16KNXN325), and the K Computer of the RIKEN Advanced Institute for Computational Science (Project ID: hp180200). This work was partly supported by MEXT as "Priority Issue on Post-K computer" (Accelerated Development of Innovative Clean Energy Systems).

References

[1] A. Fasoli, et al., Nucl. Fusion 47 S264(2007)

[2] K.L.Wong, et al., Phys. Rev. Lett. 66, 1874(1991)

[3] W.W. Heidbrink, et al., Nucl. Fusion 31, 1635 (1991)

[4] D.J. Sigmar, et al., Phys. Fluids B 41506 (1992).

[5] H.H. Duong et al., Nucl. Fusion 33, 749 (1993). 
[6] M. Isobe, et al., Nucl. Fusion 46, S918 (2006).

[7] A. Weller, et al., Phys. Plasmas 8, 931 (2001).

[8] M. Osakabe, et al., Nucl. Fusion 46, S911 (2006).

[9] K. Ogawa, et al., Nucl. Fusion 52 (2012) 094013.

[10] Y. Todo, et al., Physics of Plasmas 24, 081203 (2017)

[11] Y. Todo, M. A. Van Zeeland, A. Bierwage, and W. W. Heidbrink, Nuclear Fusion 54, $104012(2014)$.

[12] Y. Todo, M. A. Van Zeeland, A. Bierwage, W. W. Heidbrink, and M. E. Austin, Nuclear Fusion 55, 073020 (2015).

[13] Y. Todo, M. A. Van Zeeland, and W. W. Heidbrink, Nuclear Fusion 56, 112008 (2016).

[14] Y. Todo, New Journal of Physics 18, 115005 (2016).

[15] A. Bierwage and Y. Todo, Computer Physics Communications 220, 279 (2017).

[16] R. Seki, et al., Plasma Fusion Res. 5, 027 (2010).

[17] Y. Todo and T. Sato, Phys. Plasmas 5, 1321(1998).

[18] W. Park, et al., Phys. Fluids B 4, 2033 (1992).

[19] D.A. Spong, et al., Phys. Fluids B 4, 3316 (1992).

[20] Y. Todo, et al., Phys. Plasmas 2, 2711 (1995)

[21] S. Briguglio, et al., Phys. Plasmas 2, 3711 (1995) 
[22] G. Y. Fu, et al., Phys. Plasmas 13052517 (2006).

[23] X. Wang, et al., Plasma Phys. Controlled Fusion 52, 115005 (2010)

[24] R. D. Hazeltine and J.D.Meiss, Plasma confinement (Addison-Wesley Publishing Company, 1992)

[25] Y. Todo, et al., Nucl. Fusion 55, 073020 (2015)

[26] Y. Todo, et al., Nucl. Fusion 56, 112008 (2016).

[27] R.R. Mett and S. M. Hahajan, Phys. Fluids B 4, 2885 (1992).

[28] K. Harafuji, et al., J. Comput. Phys 81169 (1989)

[29] Y. Suzuki,et al., Nucl. Fusion 46, L19 (2006).

[30] D. Sarafyan, J. Math, Anal. Appl. 40, 436 (1972).

[31] T. Watanabe, Trans. Jpn. Soc. Ind. Appl.Mat. 1, 101 (1991)[in Japanese].

[32] R. Seki et al., Nucl. Fusion 53063016 (2013).

[33] D.A. Spong, et al., Phys. Plasmas 10 3217(2003)

[34] R. Seki et al., Plasma Fusion Res. 3, 016 (2008). 